\title{
Ocoxin oral solution demonstrates antiviral properties in cellular models
}

\author{
ATANASIO PANDIELLA ${ }^{1,2}$, EDUARDO SANZ ${ }^{3}$ and ELENA DÍAZ-RODRÍGUEZ ${ }^{1,2}$ \\ ${ }^{1}$ Institute of Molecular and Cellular Cancer Biology, Cancer Research Institute of Salamanca, \\ Spanish Research Council-Institute for Biomedical Research of Salamanca; \\ ${ }^{2}$ Spanish Cancer Research Biomedical Network (CIBERONC), 37007 Salamanca; ${ }^{3}$ Catalysis S.L., 28016 Madrid, Spain
}

Received March 4, 2021; Accepted June 28, 2021

DOI: $10.3892 /$ etm.2021.10561

\begin{abstract}
Ocoxin Oral Solution (OOS) and Viusid (VS) are nutritional supplements that include several natural products which affect different cellular functions, such as proliferation or the redox status. In addition, some of their constituent components have been described to exert an antiviral effect. Considering this, it was hypothesized that treatment with OOS and VS could protect from viral infections. In order to evaluate the impact of OOS and VS on viral infection, lentivirus and retrovirus whose genomes coded for green fluorescent protein were used. In addition, and as a second approach to measure viral infection, a hemagglutinin-tagged form of the mitogen-activated protein kinase ERK5 was also inserted in the retroviral vector. Viral particles produced in $293 \mathrm{~T}$ cells were used to infect HeLa cells in the presence or absence of OOS or VS. It was observed that VS had a minimal effect on the capacity of either lentivirus or retrovirus to infect HeLa cells. However, OOS significantly reduced the infection of HeLa cells with both of these viruses. The effect was dose-dependent, reaching a maximum at a 1:100 dilution of OOS. These results suggested that, in addition to its well-known antitumoral properties, OOS may also inhibit infection with viruses. This effect is relevant since patients receiving oncological therapies are more susceptible to viral infections, and nutritional supplements such as OOS may help in reducing the severity of these potential pathogenic infections.
\end{abstract}

\footnotetext{
Correspondence to: Dr Elena Díaz-Rodríguez or Dr Atanasio Pandiella, Institute of Molecular and Cellular Cancer Biology, Cancer Research Institute of Salamanca, Spanish Research Council-Institute for Biomedical Research of Salamanca, Campus Miguel de Unamuno s/n, 37007 Salamanca, Spain

E-mail: ediaz@usal.es

E-mail: atanasio@usal.es
}

Key words: nutritional supplement, viral infection, antiviral, Ocoxin, Viusid

\section{Introduction}

A strong immune system is a key component to efficiently fight both internal problems, such as the proliferation of abnormal tumor cells, as well as external insults, such as bacterial or viral infections $(1,2)$. Thus, in order to be ready to fight such external offenses more efficiently, an adequately fitted immune system is absolutely needed. Ocoxin Oral Solution (OOS) and Viusid (VS) are nutritional supplements that include several plant and natural products with an ample spectrum of biological activities $(3,4)$. Some of these products have antioxidant, vitaminic, or antiproliferative properties. Moreover, several of the constituents of these nutritional supplements have been reported to stimulate the immune system $(3,4)$.

VS composition includes several antiviral agents, antioxidants and anti-free radicals potentially able to boost the defenses of the immune system (4). Its formulation comprises licorice extract, several vitamins such as ascorbic acid or B group vitamins or amino acids (5). This combination has demonstrated to improve clinical outcomes in several scenarios, such as patients with chronic hepatitis $\mathrm{C}$ that did not respond to standard antiviral treatments or interferon therapy $(4,6)$, cirrhotic patients who have failed to achieve sustained virological response with standard of care treatments (5) or in combination with diet and exercise, to improve fatty liver disease (7). However, the mechanism of action of VS has not been fully elucidated, and research in this direction could potentiate its application to different pathologies.

OOS formulation includes plant extracts from green tea, cinnamon or licorice as well as ascorbic acid, B vitamins or several amino acids. Some of these products have been shown to exert an antitumoral activity in several experimental models (3). As a result, OOS exhibits antitumoral activity in breast, lung or colon cancer, among others [reviewed in (3), as well as (8-14)]. The molecular mechanism of action of OOS has been described to include both a decrease in cell proliferation, as well as an increase in cell death $(3,15)$. The overall effect of OOS is therefore a decrease in tumoral cell numbers. Molecularly, OOS has been reported to provoke cell cycle blockade mainly acting by increasing p27 levels, as well as by modulating the retinoblastoma pathway (15).

Current antitumoral agents, mostly those with chemotherapeutic properties, are known to affect the immune system, 
impairing it and, therefore, favoring infections $(16,17)$. The actual scenario of the COVID-19 pandemic worsens such situations as immunocompromised patients appear particularly susceptible to the physiopathological consequences of SARS-CoV-2 infection $(18,19)$. Therefore, supplements that may fight the infectivity of viruses should report benefits to patients under chemotherapeutic regimens or on those tumor types directly associated to viral infections, such as papillomavirus or hepatitis virus-promoted tumors $(20,21)$.

In the present study, it was hypothesized that OOS and VS could exhibit antiviral properties. Such possibility was evaluated in vitro on experimental preclinical models. Using flow cytometry and western blot analysis it was demonstrated that OOS, and to a lesser degree, VS, could reduce the infection of epithelial cells with retrovirus or lentivirus.

\section{Materials and methods}

Reagentsand antibodies.Cell culture media,fetalbovine serum, antibiotics and trypsin were purchased from Life Technologies. Polybrene was obtained from Sigma-Aldrich (Merck KGaA), the Immobilon P membranes from EMD Millipore and the JetPEI $^{\mathrm{TM}}$ reagent from Polyplus-transfection SA. OOS and VS were provided by Catalysis S.L. Detailed description of the components of OOS has been recently reported (3) and includes plant extracts (Glycyrrhiza glabra, Camellia sinensis and Cinnamomum verum J. Presl. extract), vitamins (ascorbic acid, pyridoxine, cyanocobalamin, folic acid and calcium pantothenate), amino acids (glycine, arginine and cysteine) and sugars (sucralose and glucosamine). As for the VS formulation, it is composed of malic acid, glycyrrhizic acid, glucosamine, arginine, calcium pantethonate, ascorbic acid, folic acid, cyanocobalamine, zinc sulfate and pyrodoxal (6). Other generic chemicals were purchased from Sigma-Aldrich, Merck KGaA. The anti-hemagglutinin (HA) monoclonal antibody (12CA5; cat. no. 11583816001) was from Roche Diagnostics and the anti-calnexin antibody (cat. no. ADI-SPA-860) from Enzo Life Sciences, Inc. The goat anti-mouse horseradish perosidase-conjugated secondary antibody (cat. no. 170-6516) was purchased from Bio-Rad Laboratories.

Cell culture, transfection, viral preparation and infection.293T, H460, OVCAR-8 and HeLa cells were grown in Dulbecco's Modified Eagle Medium supplemented with $10 \%$ fetal bovine serum and antibiotics. Cells were cultured at $37^{\circ} \mathrm{C}$ in a humidified atmosphere of $5 \% \mathrm{CO}_{2} / 95 \%$ air. 293T, HeLa and OVCAR-8 cells were usually passaged when confluent, at a 1:10 ratio and $\mathrm{H} 460$ at a 1:12 ratio. 293T, H460 and HeLa cells were obtained from the American Type Culture Collection (ATCC) and OVCAR-8 from Dr Faustino Mollinedo (Center for Biomedical Research, Madrid, Spain) who obtained them from the ATCC.

For the generation of viral particles, $2.5 \times 10^{6} 293 \mathrm{~T}$ cells were transfected using the JetPEI ${ }^{\mathrm{TM}}$ reagent following the manufacturer's instructions. To produce lentiviruses, the transfection mix containing the pLKO-GFP plasmid $(8 \mu \mathrm{g})$, as well as the packaging vectors pMDLg/RRE, pRSV-Rev and pMD2.G plasmids ( $4 \mu \mathrm{g}$ each) and the JetPEI ${ }^{\mathrm{TM}}$ reagent $(50 \mu \mathrm{l})$, were incubated for $30 \mathrm{~min}$ at room temperature prior to addition to the cells, which were maintained for $12 \mathrm{~h}$ at $37^{\circ} \mathrm{C}$, as previously described (22). The lentivirus-containing supernatants were filtered $48 \mathrm{~h}$ after transfection and used to infect HeLa, $\mathrm{H} 460$ or OVCAR-8 cells by incubation at $37^{\circ} \mathrm{C}$ in the presence of $6 \mu \mathrm{g} / \mathrm{ml}$ polybrene and the corresponding nutritional supplement (OOS or VS, at dilutions that ranged from $1: 2,000$ to $1: 100)$. After $24 \mathrm{~h}$ of incubation, media containing viral supernatants were replaced by fresh culture media in which none of the supplements was added, and $48 \mathrm{~h}$ later, samples were used for subsequent experiments.

Similarly, to generate retroviruses, either $5 \mu \mathrm{g}$ pLZR-IRES-GFP or the pLZR-HA-ERK5 were co-transfected with the retroviral accessory plasmids pMDG-VSV $(2,5 \mu \mathrm{g})$ and pNGUL-MLV-gag-pol (3 $\mu \mathrm{g})$ as previously described (23), and viral supernatants were collected and used as in the lentiviral transduction.

All the plasmids used for transfection were obtained from Addgene, Inc., collaborators (Dr A. Bernard, National Center of Biotechnology, Madrid, Spain) or prepared in the laboratory (pLZR-HA-ERK5).

Flow cytometry and microscopy. To determine the percentage of infected $\left(\mathrm{GFP}^{+}\right)$populations, cells were detached by trypsin treatment, washed twice in PBS and acquired using an Accuri C6 Flow Cytometer (BD Biosciences). A total of 20,000 events were collected for each sample and analyzed using the C6 (version 1.0.264.21) software (BD Biosciences). For each experimental condition and time-point, three independent wells were analyzed. Before the cytometric analysis, cells were observed under a fluorescence microscope, and photomicrographs were acquired using the EVOS Floid Cell Imaging Station (Life Technologies).

Preparation of cell lysates and western blot analysis. Cells were washed with PBS and lysed, as described previously (23). Briefly, cells were washed with cold PBS and lysed in ice-cold lysis buffer (140 mM NaCl; 50 mM EDTA; $10 \%$ glycerol; $1 \%$ Nonidet P-40; 20 mM Tris-HCl, pH 7.0; 1 mM PMSF; $1 \mathrm{mM} \mathrm{Na} \mathrm{O}_{4} \mathrm{~V} ; 1 \mu \mathrm{M}$ pepstatin; $1 \mu \mathrm{g} / \mathrm{ml}$ aprotinin; $1 \mu \mathrm{g} / \mathrm{ml}$ leupeptin; $25 \mathrm{mM} \beta$-glycerolphosphate; $10 \mathrm{mM} \mathrm{NaF} ; 1 \mathrm{mM}$ sodium orthovanadate), centrifuged at $4^{\circ} \mathrm{C}$ at $15,000 \mathrm{x}$ g for $10 \mathrm{~min}$, and the supernatants were transferred to new tubes. Then, $50 \mu \mathrm{g}$ total protein per lane was resolved by SDS-PAGE on $6 \%$ gels, transferred to PVDF membranes, blocked for at least $1 \mathrm{~h}$ in blocking buffer $(140 \mathrm{mM} \mathrm{NaCl}, 10 \mathrm{mM}$ Tris- $\mathrm{HCl}$, $\mathrm{pH} 7.5,0.05 \%$ Tween-20, 1\% BSA) at room temperature, and then incubated overnight at $4^{\circ} \mathrm{C}$ with the primary antibodies diluted 1:10,000 (anti-HA) or 1:30,000 (anti-calnexin). After extensive washing, the membranes were incubated for $30 \mathrm{~min}$ at room temperature with horseradish peroxidase-conjugated goat anti-mouse antibody diluted 1:10,000. Bands were visualized using a luminol-based detection system with $p$-iodophenol enhancement (Clarity Max ${ }^{\mathrm{TM}}$ Western ECL Substrate; Bio-Rad Laboratories, Inc.). Protein content was determined using a BCA assay, as previously described (23). Densitometrical analysis of the gels was performed with Image Lab Touch Software, version 2.4.0.03 (Bio-Rad Laboratories, Inc.).

Statistical analysis. Each condition was analyzed in triplicate and data are presented as the mean \pm SD of at least three independent experiments. One-way ANOVA was used to compare more than two groups. Tukey's post hoc test or Games-Howell's 
A

Pre-treatment

(24 h)

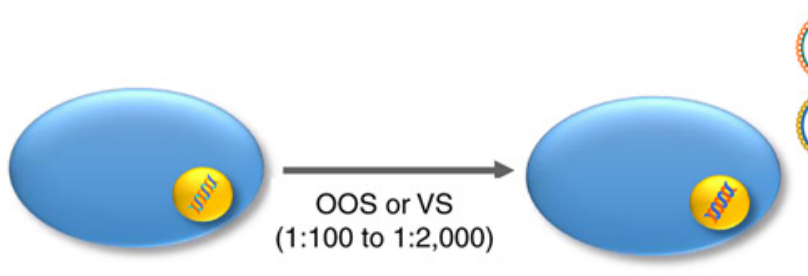

B

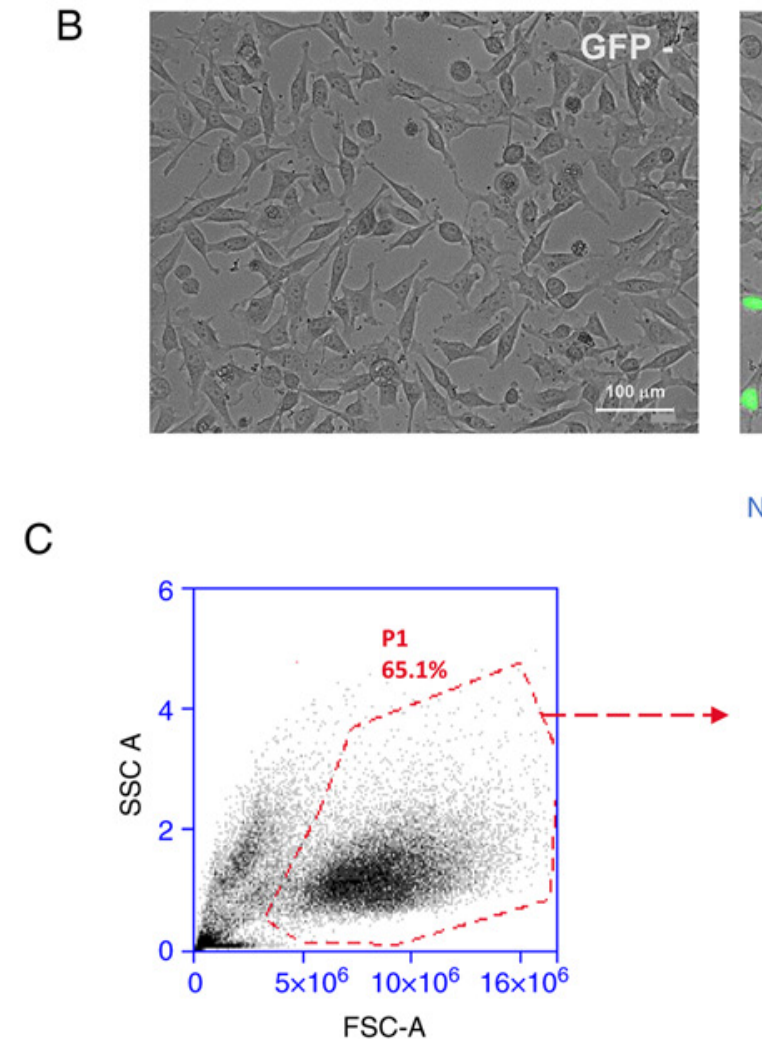

C

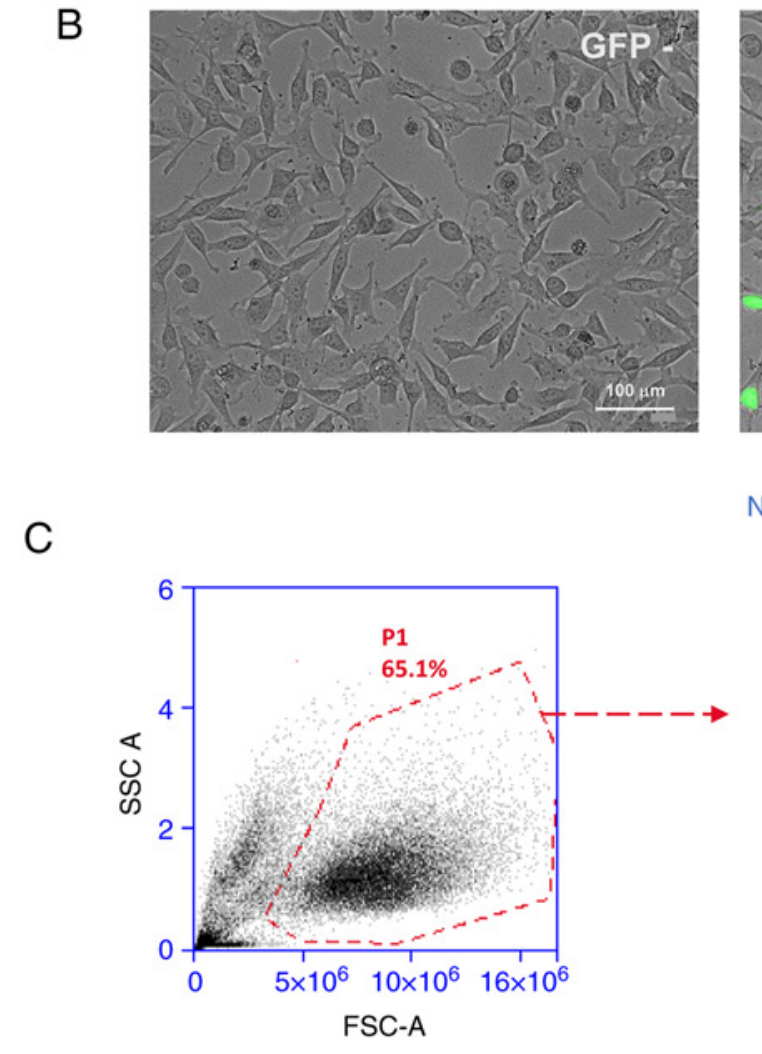

Infection

(24 h)

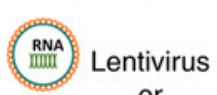

or

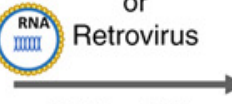

oOs or vs
Recovery

(48 h)
Detection

Cytometry

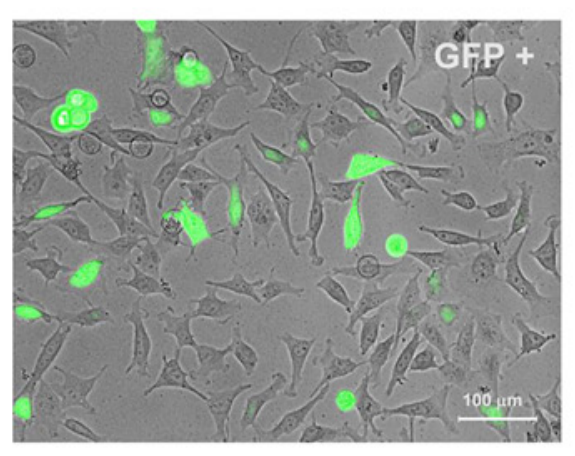

Fresh media
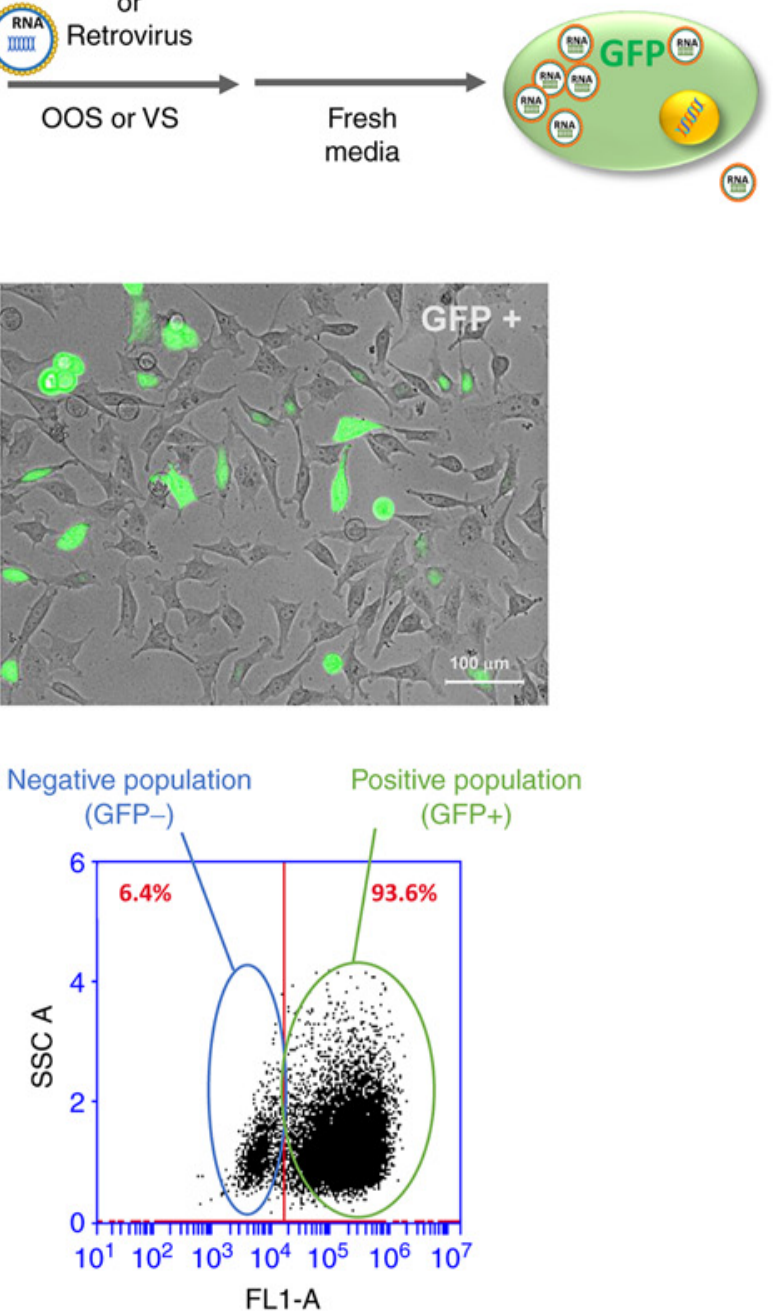

Figure 1. Representation of the experimental design. (A) Schematic representation of the experimental design. HeLa cells were pre-treated for $24 \mathrm{~h}$ with the indicated compounds, before their transduction with retro or lentiviruses, which was carried out for another $72 \mathrm{~h}$. After transduction, the incidence of transduced cells was determined by flow cytometry or western blotting. (B) Viral infection could be followed by fluorescence microscopy, since the viral particles express GFP, visible in those conditions. Scale bar, $100 \mu \mathrm{m}$. (C) Follow up of infection by flow cytometry. FSC-A and SSC-A indicate the population of living cells ( $\mathrm{P} 1$ gate). Of those cells included in P1, infected $\left(\mathrm{GFP}^{+}\right)$cells are identified, and their frequency evaluated. OOS, Ocoxin oral solution; VS, Viusid; GFP, green fluorescence protein; SSC, side scatter; FSC, forward scatter.

post hoc test were used in case of variance homogeneity or heterogeneity, respectively. Data distributions were checked for normality using the Shapiro-Wilk test, and homogeneity of variances was verified by the Levene test. $\mathrm{P}<0.05$ was considered to indicate a statistically significant difference.

\section{Results}

With the aim of testing the potential antiviral protection conferred by OOS or VS, an experimental system in which HeLa cells were infected with two types of RNA virus (lentivirus and retrovirus) was used (Fig. 1A). Both viral genomes included a coding sequence for GFP. GFP fluorescence was used to follow up the infection burden by microscopy (Fig. 1B) or flow cytometry (Fig. 1C). Infections were carried out as described in the Materials and methods section, using viral supernatant generated in $293 \mathrm{~T}$ cells and based in the retroviral vector pLZR-IRES-GFP or the lentiviral vector pLKO-GFP $(22,23)$. Microscopic analyses verified that infection with the retroviral particles induced GFP expression in a substantial number of cells (Fig. 1B). Moreover, flow cytometry showed that both vectors gave infection efficiencies as high as $90 \%$ (Fig. 1C).

To determine the capacity of OOS or VS to protect from viral infection, HeLa cells were incubated for $24 \mathrm{~h}$ with different dilutions of these products (1:100-1:2,000, schematized in Fig. 1A), then exposed to the lentiviruses in culture media containing the indicated amounts of the corresponding products. Infections in the absence and presence of the products proceeded for $24 \mathrm{~h}$ and analyzed $72 \mathrm{~h}$ post-transduction. Two control groups were included: Uninfected cells (control) and infected cells without pre-treatment (untreated positive control to which the OOS or VS-treated samples were compared). Fig. 2A shows bright 
A

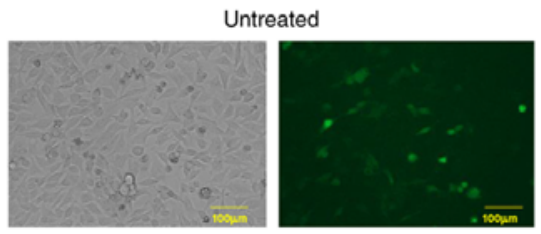

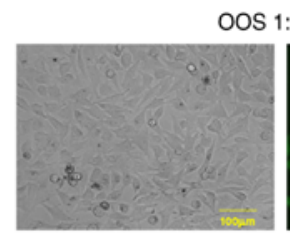

OOS 1:100

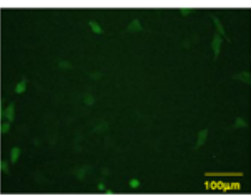

VS $1: 100$

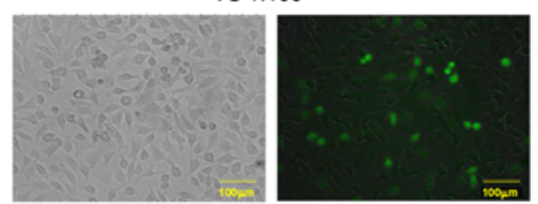

B

Infected and pre-treated as indicated

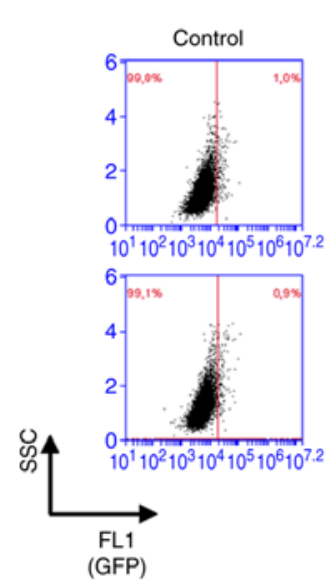

C

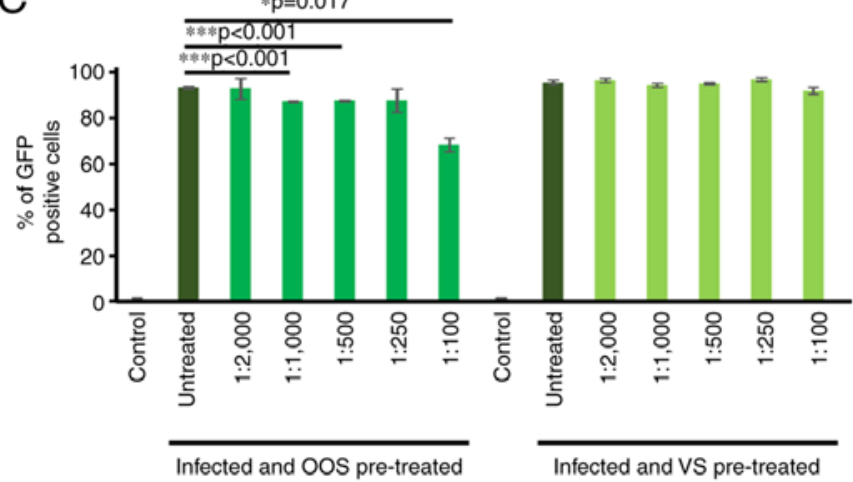

D

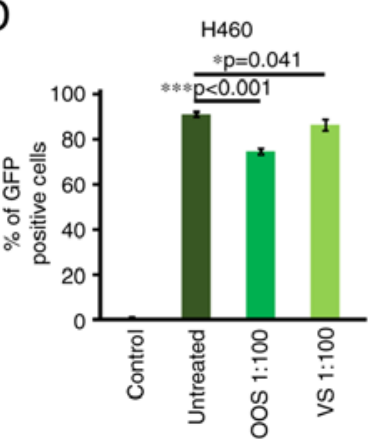

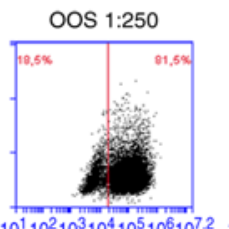

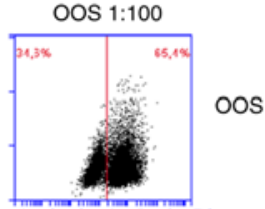

$10^{1} 10^{2} 10^{3} 10^{4} 10^{5} 10^{6} 10^{7.2} \quad 10^{1} 10^{2} 10^{3} 10^{4} 10^{5} 10^{6} 10^{7.2} 10^{1} 10^{2} 10^{3} 10^{4} 10^{5} 10^{6} 10^{7.2}$
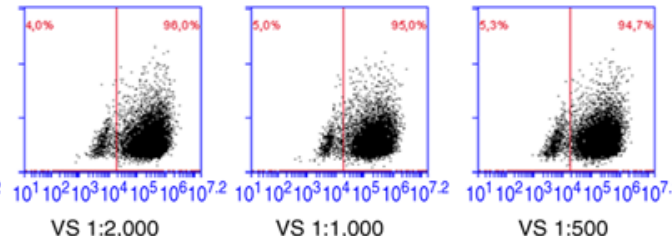

VS $1: 500$

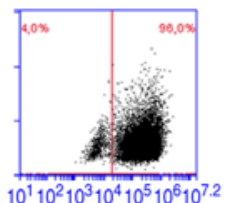

VS $1: 250$
$10^{1} 10^{2} 10^{3} 10^{4} 10^{5} 10^{6} 10^{7.2}$

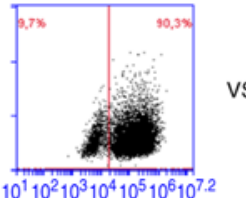

VS $1: 100$

E

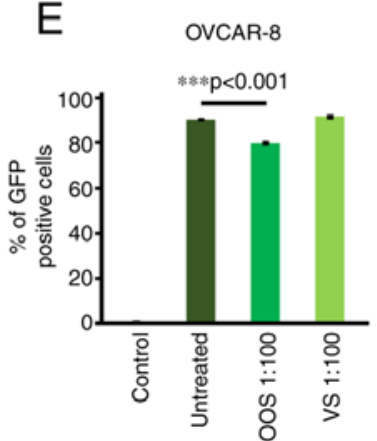

Figure 2. Antiviral effect of OOS measured by flow cytometry. (A) OOS pre-treatment and to a lesser extent VS pre-treatment, prevents cell infection by GFP-expressing lentiviruses, as shown by fluorescence microscopy. For each field, both phase-contrast (left) and fluorescence (right) images were acquired.

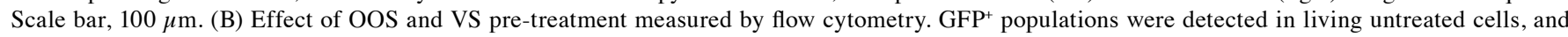
compared with those that had been pre-treated with the indicated amounts of OOS or VS. Uninfected cells were used as the negative control to establish negativity (GFP population). (C) Graphic chart of a representative experiment that was repeated 5 times. The antiviral effect of OOS and VS at a 1:100 dilution was also analyzed in (D) H460 and (E) OVCAR-8 cells in three independent experiments. ${ }^{*} \mathrm{P}<0.05$, ${ }^{* * *} \mathrm{P}<0.001$. OOS, Ocoxin oral solution; VS, Viusid; GFP, green fluorescence protein.

field, as well as fluorescence analysis, of control HeLa cells (left panels), or infected and pre-treated with OOS (middle panels) or VS (right panels) both at a 1:100 dilution in culture media. Treatment with OOS slightly decreased the number of cells $\mathrm{GFP}^{+}$cells, which was verified by flow cytometry. Under these conditions, the control cells did not express GFP and were used to establish the $\mathrm{GFP}^{+}$population (Fig. $2 \mathrm{~B}$; control). In untreated HeLa cells a $\mathrm{GFP}^{+}$population could be clearly identified and included $>90 \%$ of the cells $(95.5 \pm 1.1 \%$; Fig. $2 \mathrm{~B}$, infected and untreated). Pre-treatment with OOS decreased the infection by the lentivirus (Fig. 2B and C). Moreover, the observed antiviral effect was dose-dependent, reaching its maximal inhibition at the dilution of $1: 100(68.2 \pm 3.1 \%$; Fig. $2 \mathrm{~B}$ and C). VS induced a much milder effect, which was only detectable at the 1:100

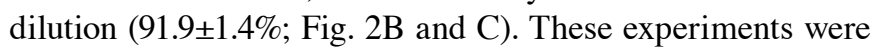
also carried out using retroviral instead of lentiviral infections, with similar results (data not shown). To determine whether this protective effect on viral infection was unique to HeLa cells or could be more general, H460 (Fig. 2D) or OVCAR-8 (Fig. 2E) cells were pre-treated with OOS or VS (both diluted 1:100) for $24 \mathrm{~h}$ prior to viral infection. In both cases, OOS significantly reduced viral infection, from $89.2 \pm 1.08$ to $73.6 \pm 1.36 \%$ in $\mathrm{H} 460$ cells (Fig. 2D and S1A) and from $91.4 \pm 0.26$ to $80.47 \pm 0.47 \%$ in OVCAR-8 cells (Fig. 2E and S1B). VS had a much milder effect, which was only detectable in H460 cells $(89.2 \pm 1.08$ to $84.6 \pm 2.46 \%$ ).

In the case of HeLa cells transduced with the pLZR-IRES-GFP retroviral vector, an alternative approach using western blot analysis was used to detect viral infection (Fig. 3A and B). To that end, since the vector includes an internal ribosomal entry site, the sequence coding for an HA-tagged version of the human mammalian mitogen-activated protein 


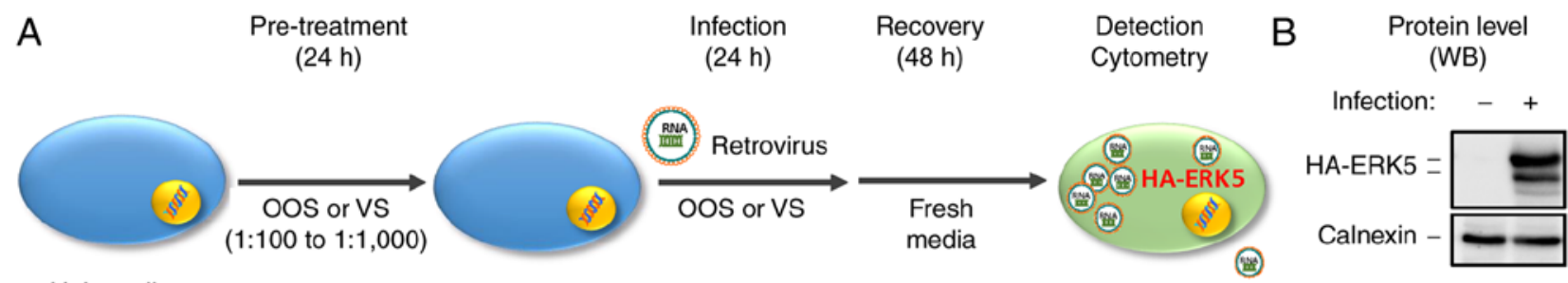

HeLa cells
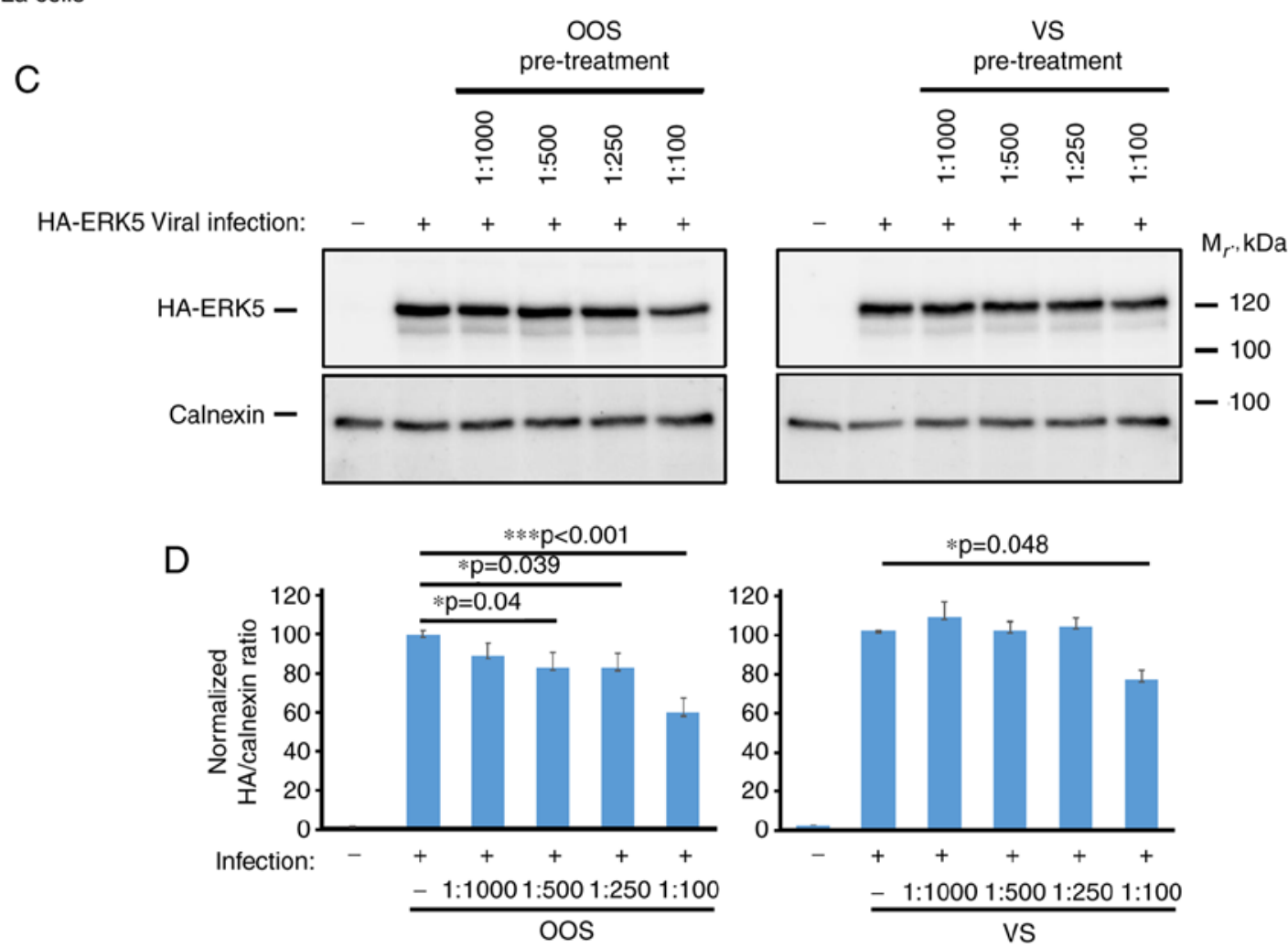

Figure 3. Antiviral effect of OOS measured by biochemistry. (A) Schematic representation of the experiments in which viral infection was measured by biochemical determination of the amount of an HA-tagged protein in western blots. (B) HA protein determination was carried out by conventional western blotting with an anti-HA antibody and calnexin was used for protein normalization. (C) OOS and VS pre-treatment prevented retroviral infection as shown in the western blot analysis with the anti-HA antibody. Membranes were reprobed with an anti-calnexin antibody that was used as a loading control for protein normalization. (D) Quantitation of HA levels in the experiment shown in panel C. The graph represents the mean \pm SD of three independent experiments. ${ }^{*} \mathrm{P}<0.05,{ }^{* * *} \mathrm{P}<0.001$. OOS, Ocoxin oral solution; HA, hemagglutinin.

kinase ERK5 was inserted. HeLa cells were plated and pre-treated with OOS or VS as aforementioned, then infected with viral particles containing pLZR-IRES-GFP-HA-ERK5. Protein lysates were prepared three days later and quantitated, and equal amounts of protein resolved by SDS-PAGE. Separated proteins in gels were transferred to PVDF membranes which were probed with the anti-HA antibody. As shown in Fig. 3B and C, the anti-HA antibody failed to recognize any band in the uninfected HeLa cells. In contrast, in cells transduced with the retrovirus, the anti-HA antibody strongly reacted with a $120 \mathrm{kDa}$ protein, which corresponded to HA-tagged ERK5. In this context, pre-treatment with both OOS and VS prevented the infection with pLZR-HA-ERK5 in a dose-dependent manner, reaching an inhibition rate of up to $40 \pm 1.4$ and $24.8 \pm 4.9 \%$, respectively. In this experimental setting, OOS also demonstrated a much stronger effect than that caused by VS (Fig. 3C and D). Reprobing of the blots with an antibody to calnexin was used to verify equal amounts of protein loading as well as to use that protein as an internal standard to normalize expression amounts (Fig. 3D).

\section{Discussion}

In the present study, the potential antiviral activities of the nutritional supplements OOS and VS were investigated, demonstrating that OOS pre-treatment was able to reduce both retroviral and lentiviral infection. OOS has demonstrated its antitumoral activity in several clinical trials including hepatocellular carcinoma (24), melanoma (25), head and neck (26), prostate (27), cervical and endometrial cancer (28) among others (29,30), and can improve the quality of life of patients (3). On the other hand, VS is a nutritional supplement that has properties as immunomodulator and hepatoprotector $(4,5,7,31,32)$ which have also been tested in a number of trials (31-33).

OOS and VS are formulations that include several compounds with anti-oxidant, anti-inflammatory and antiviral properties (3). These properties make those products attractive to help in fighting different diseases. Given the fact that some of the constituent components of OOS and VS have been reported to act as antiviral compounds, the aim of the present study was to further confirm whether OOS or VS could have antiviral 
properties. Such study is important, since patients who receive OOS or VS suffer from diseases in which the immune system may be compromised, and therefore are more susceptible to infections. Such is the case of oncological disorders $(16,17)$. In pathologies such as leukemia, glioblastoma, hepatocellular carcinoma, or breast, lung, prostate, colon or pancreatic cancer, OOS has been shown to reduce the proliferation of a number of cell lines and xenografted tumors in mice (8-14). Moreover, several clinical studies have indicated that this product is not toxic and may favor quality of life of patients, supporting its use as a nutritional supplement $(24,27,29,30)$. In case that OOS or VS would exhibit antiviral capabilities, they should be considered for its use in the treatment and prevention of other cancer types that are known to be caused or associated to viral infections, such as non-Hodgkin's lymphoma, or gastric, hepatic or cervical cancer, among others (20,21,34-36).

In the present study, OOS and VS exerted antiviral effects, as demonstrated by their ability to reduce viral infection caused by two types of RNA viruses. This effect was evidenced by a reduction in GFP fluorescence in several cell lines infected with retroviral or lentiviral vectors, as well as a decreased production of retrovirus-derived HA-ERK5. The date indicated that, while the antiviral effect of OOS was larger than that of VS, such effect was far from being high. Although the effect of these compounds could have been potentiated by increasing its concentration, this option was discarded because this higher concentration may affect not only viral infection, but also cell proliferation as happens in several cellular models (8-11), making the effects more difficult to interpret. Nevertheless, while the antiviral benefit may be small it may be useful to decrease viral infective capacity and therefore increase the body's defense mechanism against infections. Given the special susceptibility of some patients to viral infections, the fact that OOS or VS may have antiviral properties, in addition to their immunostimulatory effects, appears an attractive property that should further evaluated.

Finally, the actual threat that the COVID-19 pandemic represents for oncological patients also needs to be considered $(18,19)$. Given the discrete but reproducible antiviral effect of VS and especially OOS, and the fact that those products have demonstrated to be safe and well-tolerated in several clinical contexts $(3-7,37)$, they may be considered in the fight against SARS-CoV-2 infections. Furthermore, several recent reports indicate that some of the constituents of OOS and VS may be effective at counteracting COVID-19 both reducing the infectivity or severity of the disease $(8-11,38-40)$. Besides the use of food supplements with antioxidant and anti-inflammatory properties seems to be beneficial in that context $(41,42)$. In fact, clinical trials in that direction, evaluating the antiviral protective actions of VS are currently ongoing (https://www. clinicaltrials.gov/ct2/show/NCT04407182), while others have recently been published $(43,44)$. However, the exact mechanism through which OOS or VS cause their antiviral effect is unknown, although these products could affect viral entry or replication. The present study suggested that exploration of the antiviral actions of OOS is worthwhile, and may help understand whether this supplement could help prevent infections in oncological patients whose immune system is immunocompromised.

\section{Acknowledgements}

The authors would like to acknowledge the Pandiella group (Cancer Research Center of Salamanca, Spain) for their helpful discussions and continuous support, and specially Ms. L. Gandullo, for her help with the statistical analysis.

\section{Funding}

AP is a staff employee of the Spanish Research Council (CSIC) and EDR is supported by a postdoctoral contract from the same institution. ES is an employee of Catalysis S.L. The work on ocoxin oral solution in preclinical models carried out by $\mathrm{AP}$ and EDR has received partial support from Catalysis S.L.

\section{Availability of data and materials}

The datasets used and/or analyzed during the current study are available from the corresponding author on reasonable request.

\section{Authors' contributions}

EDR carried out the experiments. Both EDR and AP designed the research, analyzed the experiments, wrote the paper and prepared the figures. ES assisted in data analysis and manuscript preparation. All authors read and approved the final manuscript. AP and EDR confirm the authenticity of all the raw data.

\section{Ethics approval and consent to participate}

Not applicable.

\section{Patient consent for publication}

Not applicable.

\section{Competing interests}

ES is an employee of Catalysis S.L. The work on OOS in preclinical models carried out by AP and EDR has received partial support from Catalysis S.L.

\section{References}

1. McCombS, Thiriot A,Akache B, Krishnan Land Stark F: Introduction to the immune system. Methods Mol Biol 2024: 1-24, 2019.

2. Parkin J and Cohen B: An overview of the immune system. Lancet 357: 1777-1789, 2001

3. Pandiella-Alonso A, Diaz-Rodriguez E and Sanz E: Antitumoral properties of the nutritional supplement ocoxin oral solution: A comprehensive review. Nutrients 12: 2661, 2020.

4. Gomez EV, Perez YM, Sanchez HV, Forment GR, Soler EA, Bertot LC, Garcia AY, del Rosario Abreu Vazquez M and Fabian LG: Antioxidant and immunomodulatory effects of Viusid in patients with chronic hepatitis C. World J Gastroenterol 16: 2638-2647, 2010.

5. Vilar Gomez E, Sanchez Rodriguez Y, Torres Gonzalez A, Calzadilla Bertot L, Arus Soler E, Martinez Perez Y, Yasells Garcia A and Abreu Vazquez Mdel R: Viusid, a nutritional supplement, increases survival and reduces disease progression in HCV-related decompensated cirrhosis: A randomised and controlled trial. BMJ Open 1: e000140, 2011. 
6. Vilar Gomez E, Gra Oramas B, Soler E, Llanio Navarro R and Ruenes Domech C: Viusid, a nutritional supplement, in combination with interferon alpha-2b and ribavirin in patients with chronic hepatitis C. Liver Int 27: 247-259, 2007.

7. Vilar Gomez E, Rodriguez De Miranda A, Gra Oramas B, Arus Soler E, Llanio Navarro R, Calzadilla Bertot L, Yasells Garcia A and Del Rosario Abreu Vazquez M: Clinical trial: A nutritional supplement Viusid, in combination with diet and exercise, in patients with nonalcoholic fatty liver disease. Aliment Pharmacol Ther 30: 999-1009, 2009.

8. Diaz-Rodriguez E, El-Mallah AM, Sanz E and Pandiella A Antitumoral effect of Ocoxin in hepatocellular carcinoma. Oncol Lett 14: 1950-1958, 2017.

9. Diaz-Rodriguez E, Hernandez-Garcia S, Sanz E and Pandiella A Antitumoral effect of Ocoxin on acute myeloid leukemia. Oncotarget 7: 6231-6242, 2016.

10. Diaz-Rodriguez E, Sanz E and Pandiella A: Antitumoral effect of Ocoxin, a natural compound-containing nutritional supplement, in small cell lung cancer. Int J Oncol 53: 113-123, 2018.

11. Hernandez-Garcia S, Gonzalez V, Sanz E and Pandiella A: Effect of oncoxin oral solution in HER2-overexpressing breast cancer. Nutr Cancer 67: 1159-1169, 2015

12. Hernandez-SanMiguel E, Gargini R, Cejalvo T, Segura-Collar B, Núñez-Hervada P, Hortigüela R, Sepúlveda-Sánchez JM, Hernández-Laín A, Pérez-Núñez A, Sanz E and Sánchez-Gómez P: Ocoxin modulates cancer stem cells and M2 macrophage polarization in glioblastoma. Oxid Med Cell Longev 2019: 9719730, 2019.

13. Hernandez-Unzueta I, Benedicto A, Olaso E, Sanz E, Viera C, Arteta B and Márquez J: Ocoxin oral solution ${ }^{\circledR}$ as a complement to irinotecan chemotherapy in the metastatic progression of colorectal cancer to the liver. Oncol Lett 13: 4002-4012, 2017.

14. Hernandez-Unzueta I, Benedicto A, Romayor I, Herrero A, Sanz E, Arteta B, Olaso E and Márquez J: Ocoxin oral solution exerts an antitumoral effect in pancreatic cancer and reduces the stromal-mediated chemoresistance. Pancreas 48 555-567, 2019.

15. Perez-Pena J, Diaz-Rodriguez E, Sanz E and Pandiella A: Central role of cell cycle regulation in the antitumoral action of ocoxin. Nutrients 11: 1068, 2019.

16. Rusu RA, Sirbu D, Curseu D, Năsui B, Sava M, Vesa ŞC Bojan A, Lisencu C and Popa M: Chemotherapy-related infectious complications in patients with Hematologic malignancies. J Res Med Sci 23: 68, 2018

17. Vento $\mathrm{S}$ and Cainelli $\mathrm{F}$ : Infections in patients with cancer undergoing chemotherapy: Aetiology, prevention, and treatment. Lancet Oncol 4: 595-604, 2003.

18. Dai M, Liu D, Liu M, Zhou F, Li G, Chen Z, Zhang Z, You H, Wu M, Zheng Q, et al: Patients with cancer appear more vulnerable to SARS-CoV-2: A multicenter study during the COVID-19 outbreak. Cancer Discov 10: 783-791, 2020.

19. Fuentes-Antras J, Manzano A, Marquina G, Paz M, Aguado C, Granja M, Benítez J, Ortega J, Priego A, González C, et al: A snapshot of COVID-19 infection in patients with solid tumors. Int J Cancer: Dec 3, 2020 (Epub ahead of print). doi: 10.1002/ ijc. 33420 .

20. Bouvard V, Baan R, Straif K, Grosse Y, Secretan B, El Ghissassi F, Benbrahim-Tallaa L, Guha N, Freeman C, Galichet L, et al: A review of human carcinogens-Part B Biological agents. Lancet Oncol 10: 321-322, 2009.

21. Fernandez AF and Esteller M: Viral epigenomes in human tumorigenesis. Oncogene 29: 1405-1420,2010.

22. Diaz-Rodriguez E and Pandiella A: Modulation of cereblon levels by anti-myeloma agents. Leuk Lymphoma 57: 167-176, 2016.

23. Diaz-Rodriguez E, Álvarez-Fernández S, Chen X, Paiva B López-Pérez R, García-Hernández JL, San Miguel JF and Pandiella A: Deficient spindle assembly checkpoint in multiple myeloma. PLoS One 6: e27583, 2011.

24. Al-Mahtab M, Akbar SM, Khan MS and Rahman S: Increased survival of patients with end-stage hepatocellular carcinoma due to intake of ONCOXIN(R), a dietary supplement. Indian J Cancer 52: 443-446, 2015

25. Gray Lovio OR, Abreu Daniel A, García Yánez LA, Rodríguez MO, González CV, Lence Anta JJ and Sanz E: Efficacy and safety of oncoxin-viusid, a nutritional supplement, in twenty patients with stage IIB-III of cutaneous melanoma: An open-label proof of concept study. J Cancer Sci Ther 11: 263-268, 2019.
26. Rivas IC, Silva JA, Alfonso G, Candanedo H, Cuervo Y, Mestre B, Mestre Cabello JR, Lence J, Lugoyo M and Sanz E: Oncoxin-viusid with radiotherapy and chemotherapy in patients with head and neck cancer: Results from a phase II, randomised, double-blind study. J Cancer Sci Ther 10: 317-327, 2018.

27. Fundora Ramos MI, Maden LB, Casanova FO, Cruz FH, Reyes CS, Gato AH, Lyncon IB, González EV, Morales KP, Lence JJ and Sanz E: Oncoxin-Viusid ${ }^{\circledR}$ may improve quality of life and survival in patients with hormone-refractory prostate cancer undergoing onco-specific treatments. Mol Clin Oncol 14: 5,2021

28. Ruiz Lorente R, Hernández Durán D, García Viamontes J, Lence Anta J, Ortiz Reyes R and Sanz E: Efficacy of Oncoxin-viusid on the reduction of adverse reactions to chemotherapy and radiotherapy in patients diagnosed with cervical cancer and endometrial adenocarcinoma. J Cancer Ther 11: 276-295, 2020

29. Uddin D, Islam MA, Mahmood I, Ghosh AK, KhatunRA and Kundu S: Findings of the 3-month supportive treatment with ocoxin solution beside the standard modalities of patients with different neoplastic diseases. TAJ 22: 172-175, 2009.

30. Kaidarova DR, Kopp MV, Pokrovsky VS, Dzhugashvili M, Akimzhanova ZM, Abdrakhmanov RZ, Babich EN, Bilan EV, Byakhov AV, Gurov SN, et al: Multicomponent nutritional supplement Oncoxin and its influence on quality of life and therapy toxicity in patients receiving adjuvant chemotherapy. Oncol Lett 18: 5644-5652, 2019.

31. Ashraf S, Alam J, Sarkar JA, Khondaker FA, Farhana Y and Khan NA: An open-label randomized clinical study to compare the effects of a nutritional supplement versus vitamin $\mathrm{E}$ on fibroscan score in nonalcoholic steatohepatitis (NASH) patients. Univ J Public Health 6: 56-62, 2018.

32. Pascal R and Russu E: 'Viusud' as a immnunomodultaor drug for the treatment of immunological disturbances in psoriatic arthritis. Arta Medica 4: 38-42, 2006

33. Valencia MH, Pacheco AC, Quijano TH, Girón AV and López CV: Clinical response to glycyrrhizinic acid in genital infection due to human papillomavirus and low-grade squamous intraepithelial lesion. Clin Pract 1: e93, 2011

34. de Martel C, Georges D, Bray F, Ferlay J and Clifford GM: Global burden of cancer attributable to infections in 2018: A worldwide incidence analysis. Lancet Glob Health 8: e180-e190, 2020.

35. Plummer M, de Martel C, Vignat J,Ferlay J, Bray F and Franceschi S: Global burden of cancers attributable to infections in 2012: A synthetic analysis. Lancet Glob Health 4: e609-e616, 2016.

36. Schelhaas M: Viruses and cancer: Molecular relations and perspectives. Biol Chem 398: 815-816, 2017

37. Dominguez Gomez J, Simon RD, Abreu Daniel A and Zelenkova H: Effectiveness of glycyrrhizinic Acid (glizigen) and an immunostimulant (viusid) to treat anogenital warts. ISRN Dermatol 2012 863692,2012

38. Bae M and Kim H: Mini-review on the roles of vitamin C, vitamin $\mathrm{D}$, and selenium in the immune system against COVID-19. Molecules 25: 5346, 2020.

39. Menegazzi M, Campagnari R, Bertoldi M, Crupi R, Di Paola R and Cuzzocrea S: Protective effect of epigallocatechin-3-gallate (EGCG) in diseases with uncontrolled immune activation: Could such a scenario be helpful to counteract COVID-19? Int J Mol Sci 21: 5171, 2020.

40. Mhatre S, Srivastava T, Naik S and Patravale V: Antiviral activity of green tea and black tea polyphenols in prophylaxis and treatment of COVID-19: A review. Phytomedicine 85: 153286, 2021.

41. Mrityunjaya M, Pavithra V, Neelam R, Janhavi P, Halami PM and Ravindra PV: Immune-boosting, antioxidant and anti-inflammatory food supplements targeting pathogenesis of COVID-19. Front Immunol 11: 570122, 2020.

42. Lammi C and Arnoldi A: Food-derived antioxidants and COVID-19. J Food Biochem 45: e13557, 2020

43. Benites W, Heras MV, Mero ML and Marquez D: Effectiveness of VIUSID ${ }^{\circledR}$ and ASBRIP ${ }^{\circledR}$ in hospitalized patients infected by SARS-CoV-2 and mild-to-moderate respiratory illness. An observational prospective study. Clin Infect Dis: 5, 2021.

44. Petrov P, Mihaylov A, Shopove M, Boncheva M and Marquez D: Efficacy and safety of viusid and asbrip in hospitalized patients with mild and moderate COVID-19: A randomized controlled trial. Adv Infectious Dis 11: 171-184, 2021.

This work is licensed under a Creative Commons Attribution-NonCommercial-NoDerivatives 4.0 International (CC BY-NC-ND 4.0) License. 